\title{
Recent Advancement and Innovations in CRISPR/Cas and CRISPR Related Technologies: A review
}

Sikandar Hayat Khan

MBBS, Fellowship in Chemical Pathology, PgD Endocrinology \& Diabetes, MSc Cancer Molecular Pathology \& Genomics, Certification in Clinical Research, Pakistan.

Corresponding author: Sikandar Hayat Khan, MBBS, Fellowship in Chemical Pathology, PgD Endocrinology \& Diabetes, MSc Cancer Molecular Pathology \& Genomics, Certification in Clinical Research, Pakistan.

Received date: May 12, 2021; Accepted date: June 07, 2021; Published date: June 24, 2021

Citation: Sikandar H. Khan, (2021) Recent Advancement and Innovations in CRISPR/Cas and CRISPR Related Technologies: A review. J, Biotechnology and Bioprocessing, 2(5); DOI: 10.31579/2766-2314/042

Copyright: (C) 2021, Sikandar Hayat Khan, This is an open access article distributed under the Creative Commons Attribution License, which permits unrestricted use, distribution, and reproduction in any medium, provided the original work is properly cited.

\begin{abstract}
CRISPR genome editing technologies have been improving by every passing day. The initial CRISPR/Cas9 technologies, though emerged an improved version of genome editing in competition with TALENS and ZFNs, was nevertheless not free from technical and off-target effects. Technological improvements overtime start addressing issues with original CRISPR/Cas9 technology. The major areas of improvement targeted nucleases and delivery methods. Overtime the nuclease like Cas9 had some modifications like FokI-dCas9, Truncated guide RNAs (trugRNAs), Paired Cas9 nickase, Cpf1, Cas6 with Csm/Csr complex and chemically treated Cas9. In terms of delivery methods the improvements came along after almost all methods including viral methods like Recombinant Adeno Associated Viruses (rAAV), Lentivirus (LV), and bacteriophages. The review summarizes various non-viral gene delivery modes including physical methods like electroporation and chemical methods like nano particles, cell-derived membrane vesicles (CMVs) with upgraded developments. The review also compares various modes of delivering CRISPR gene editing machinery.

Key words: Cluster Regularly Interspaced Short Palindromic Repeats (CRISPR), cas nuclease, viral delivery methods, Adeno-Associated Virus (AAVs), Peptide Nucleic Acids (PNAs), Cell-derived Membrane Vesicles (CMVs)
\end{abstract}

\section{Background}

Cluster Regularly Interspaced Short Palindromic Repeat (CRISPR) currently emerged as the standalone technology in the field of genome editing. While the technology is being improvised at a very fast pace by every given day, historically, the seminal discovery of "CRISPR" has attributes dating back to the 1990s when Mojica et al after a decade of research work coined the term "CRISPR" as a form of adoptive immune response among bacteriophages [1]. In nature the CRISPR/Cas system are available in various forms in Bacteria and Achaea and has been classified and sub-classified Class-1 system incorporating multiple effector operons and Class-2 system using a single effector protein [2]. Later various pieces were joined through biotechnology to shape the system i.e., CRISPR/Cas9 to edit genome by the help of a simplified guide RNA formed by fusion of tracrRNA (Trans activating CRISPR RNA) and crRNA which acting as a duplex with ability to guide Cas9 accurately to its cleavage site on DNA strand. [3] CRISPR Associated protein-9 with inherent endonuclease function assisted by "gRNA" was also able to efficiently create specific double-helical breaks in the DNA, thus further improving gene editing quality [3]. Provided the previously in vogue Zinc Finger Nucleases (ZFNs) and Transcription Activator Like Effector Nucleases (TALENs) showed much promise as gene editing options, still CRISPR technology was able provide better alternative as it did not require reengineering the enzyme for every new target sequence. $[4,5]$ Furthermore the limitations, lesser efficiency and reengineering issues with earlier genome editing techniques led to the rise of CRISPR/Cas9 system by virtue of its feasibility of engineering, cost-effectiveness and measurability [6] Though considered in early years of development of "CRISPR/Cas9 genome editing system" as a diagnostic and therapeutic panacea, it was sooner realized with biotechnological advancements, ongoing research and market competitiveness that the technique has relative shortcomings which need improvisations. The initial technology as devised by Jennifer Doudona and Emmanuelle Charpentier suffered due to multiple off-target effects including: a-chromosomal translocations and random mutations in general [7], b-immunogenicity related to Cas9 proteins, sgRNA and sometimes with inserted DNA fragments[8], c-efficiency in CRISPR/Cas payload delivery into cell [9], appearance of resistance after successful CRISPR/Cas9 use as therapeutic strategy to eradicate certain infections like HIV [10], and finally the inability of conventional CRISPR/Cas9 technology to edit PAM free sequence and sometimes CRISPR-resistant systems [11]. Thus the novel CRISPR/Cas9 technology was required to be improved. Three key areas were identified for further improvement in CRISPR technologies, including Cas9/gRNA efficiency as nucleases, overcoming size issue of Cas9 to address cellular accessibility issues and the degree of accuracy in genome editing (fidelity) [12].

Though the acknowledged limitations and gaps in perfecting the original CRISPR/Cas9 technology, innovation and biotechnological "bubble burst" phenomena is at work to overcome the various issues related with CRISPR/Cas systems. These attempts for improving the CRISPR/Cas9 
technology resulted in multiple variations in initial technologies which can be termed as the "Next generation CRISPR/Cas9 technologies" with the aim to overcome the bottlenecks for both diagnostic industry and clinical application to address micro-precision based personalized medicine. The objective of this review is to discuss the multiple biotechnological innovations, renovations and methods adopted to reduce off-target effects and improve yield of genome editing through CRISPR technologies which have recently surfaced in the clinical and research market arena.

\section{CRISPR technology}

The general concept of CRISPR/Cas technologies was taken from the archea and bacterial immune system where the system inherently works as an adoptive immune system [13]. Generally speaking the traditional concept of "CRISPR" includes two components including "CRI" and "SPR", where the former stands for "Clustered Regularly Interspaced", which are located between various spacers/SRIs. The latter category "SPR" stands for "Short Palindromic repeats" with differences in their nucleotides and act like spacers with genetic memory and to segregates the various CRIs [14]. Alongside CRISPR there are nuclease proteins termed CRISPR-associated proteins (Cas). In a typical CRISPR/Cas9 format sgRNA (crRNA +tracrRNA) guides the Cas9 nucleases to site of cleavage where the dsDNA nicks are made by nucleases. The sgRNA actually identifies the wrong coded/disease causing DNA sequence which is further cleaved by Cas9 proteins. Following removal of nondesirable DNA segment, the desirable coded sequence is inserted and this allows correction of mutation and disease in theory and in most experimental works including application on human genome [15].

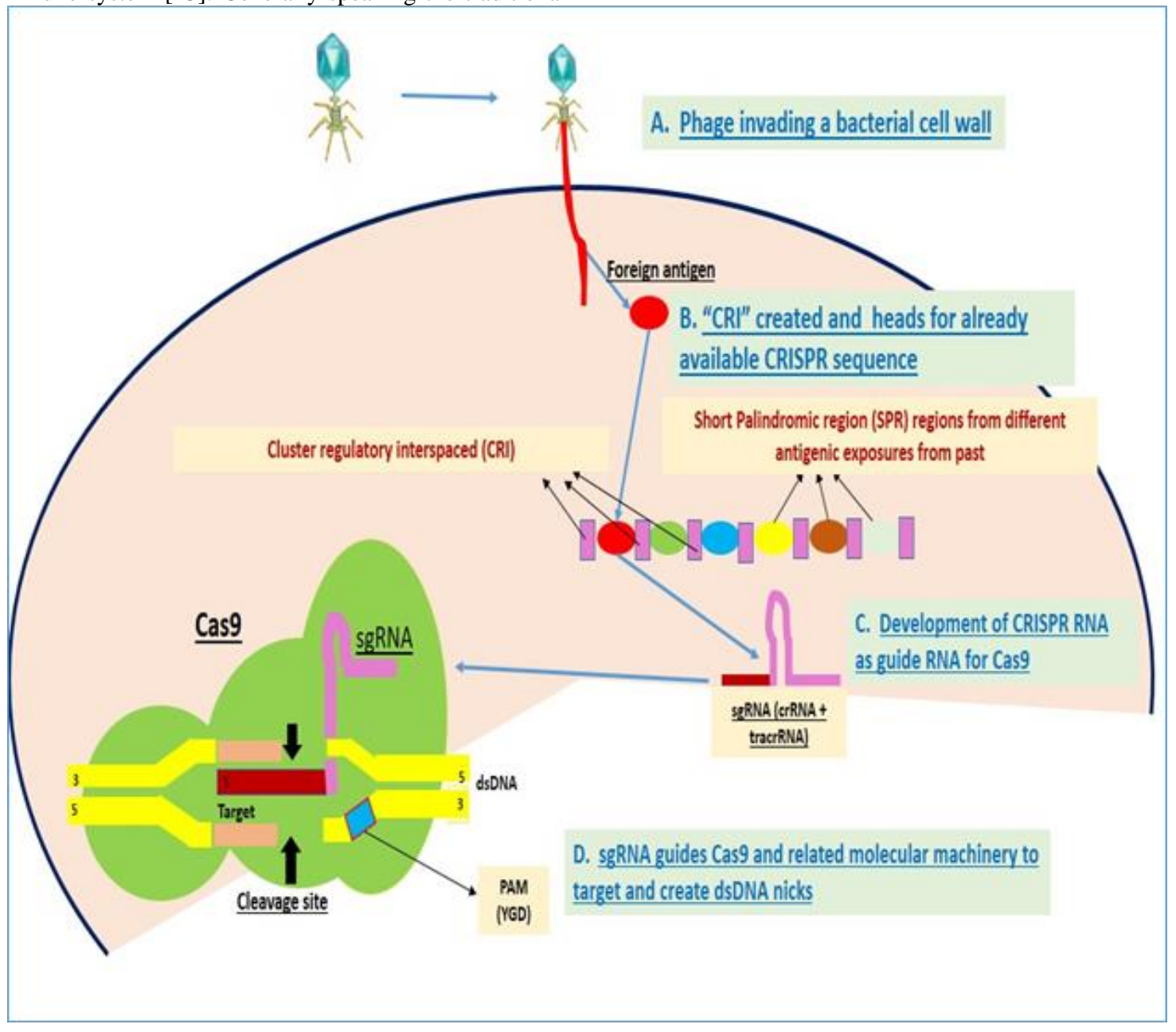

Figure-1:- Schematic showing basic step wise concept CRISPR/Cas9 technology

Although the technique in general is still almost the same but various modifications and improvisations have been introduced to reduce the associated off-target effects and to make the genome editing mode more efficient.

\section{Review methodology}

The biotechnological plight has really taken up an upsurge with multiple clinical applications on animal models, improvised versions and techniques to reduce the side effects, enhancing cellular entry efficiency and targeting better gene edited products. Over the last five years or so CRISPR technology has seen an explosion of diverse and novel developments of biotechnological tools to make the technique more efficient, less cytotoxic and high throughput modality. However, it becomes also difficult for a commoner starting the subject to grasp the multiple CIRSPR versions and sub-types with modifications. An attempt therefore is made to provide a concise overview of various CRISPR modifications, albeit with generalization due to fast increasing research on the subject both in the diagnostic and therapeutic industry. The literature search with word "CRISPR genome editing" on PubMed highlighted 5913 articles where 5-year filter generated majority of the articles $(n=5778)$ in last 5 years, highlighting the rapid focus on the technique. The research was short-listed $(n=778)$ by applying additional filter including only reviews, and studies done on animals including humans (Excluding botanical literature). Author reviewed all 778 
abstracts initially dealing with CRISPR technology. Author further narrowed down our review work to 86 articles mentioning any modification or attempting some different methodology in using CRISP technologies. Articles dealing with conventional use of CRISPR/Cas9 technology in a specific disease among animal models, using newer genome editing techniques like integrase, synthetic genomics, were also excluded. Wherever a differential technique or modification was suggested in CRISPR technology, we traced down original article to provide primary reference.

\section{CRISPR technological modifications, improvisations and innovations}

The improvements in CRISPR/Cas technology has followed multiple dimensions starting from mechanisms to enhance payload entries, addressing payload in specific for enhancing genome editing, or simplifying techniques by altering or obviating some step. All these modifications were targeted to miniaturize the technology for common laboratory usage, tailor making for certain clinical or research requirements, reducing off-target side effects and in specific to enhance efficiency of final genome edited product. A brief overview of these technological improvisations is given in figure-2. It's not practical to describe the details of all types of CRISPR technologies, but a simplistic overview with aim to introduce, explain and highlight the advantages or otherwise of these technologies is presented here.

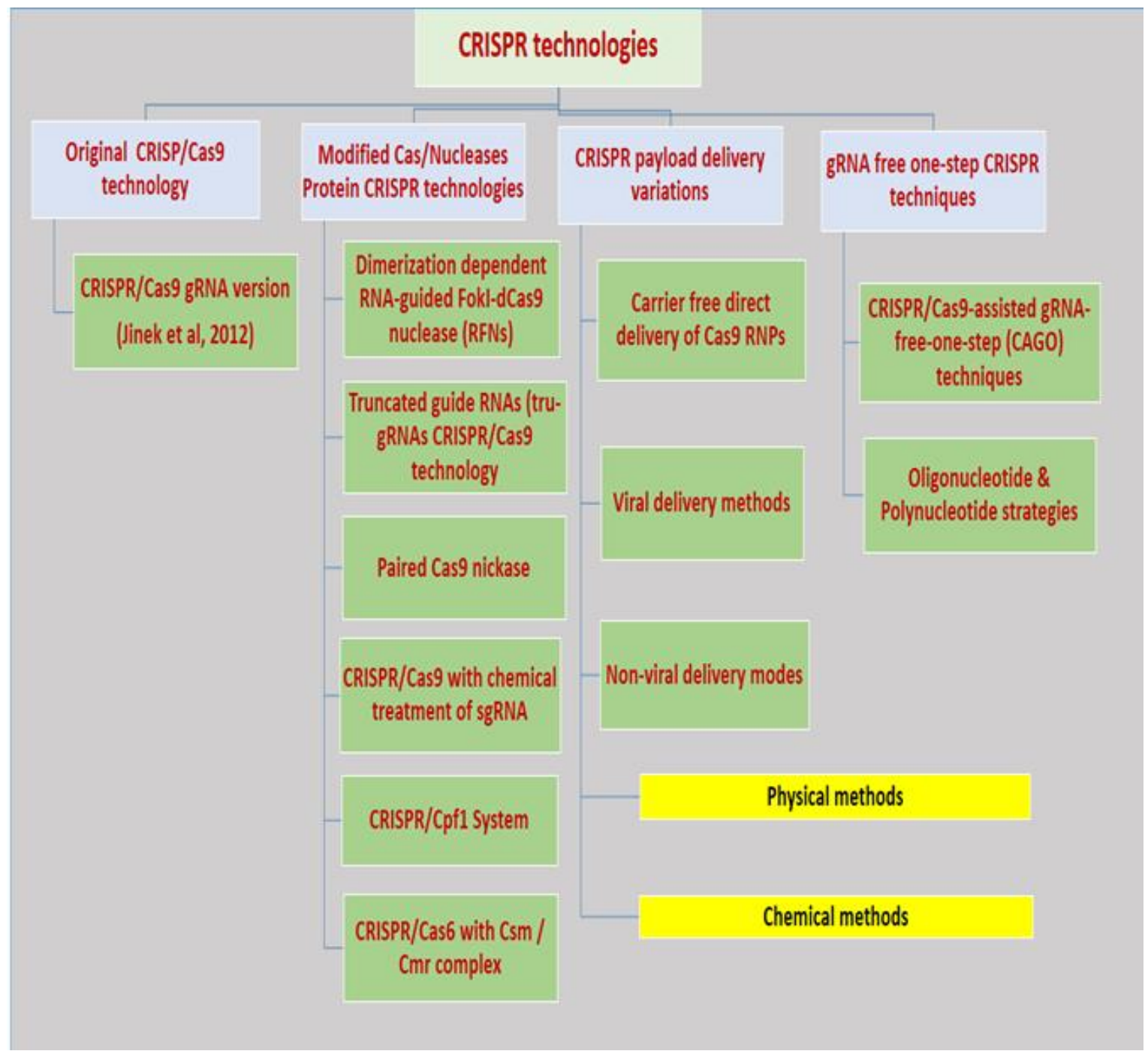

Table-1: Various modifications and improvements attempted over last few years in CRISPR/Cas technology

a. Modified Cas/Nucleases protein related CRISPR technologies: Cas 9 protein have been considered as one of the major sources of nondesirable mutagenic side-effects. These issues resulted both because of the size of the Cas9 protein and also being non-specific manipulator of Proto-spacer Adjacent Motif (PAM) including 5'-NGG-3' [16]. Poor recognition of this motif result in multiple issues leading to mutagenisis and decreased specificity ultimately affecting the editing efficiency. So improving characteristics including size of Cas9 and possible manipulation Cas9 interactions 5-end of gRNA becomes an active area for improving editing function [17]. Apart from Cas9 protein size, literature review also highlights appearance of both adoptive and cellular immunity to Cas9 along with presence of Anti-Cas9 antibodies which decreases the chances of optimal genome editing success [18].

i. Dimerization dependent RNA-guided FokI-dCas9 nuclease (RFNs): Wyvekens et al utilized combination approach where they created a tru-RFNs (Truncated-gRNAs +Dimerization dependent RNA-guided FoKI-dCas9 nucleases termed RFNs). This approach not only reduces the off-target mutagenesis (OTMs) resulting from FokI-dCas9 along with increase in gene editing specificity due to modification of dCas9 [19]. The technique resulted in reduced off- 
target mutagenesis and cytotoxicity with improved efficiency. The overall explanation of technique is provided in figure-3.

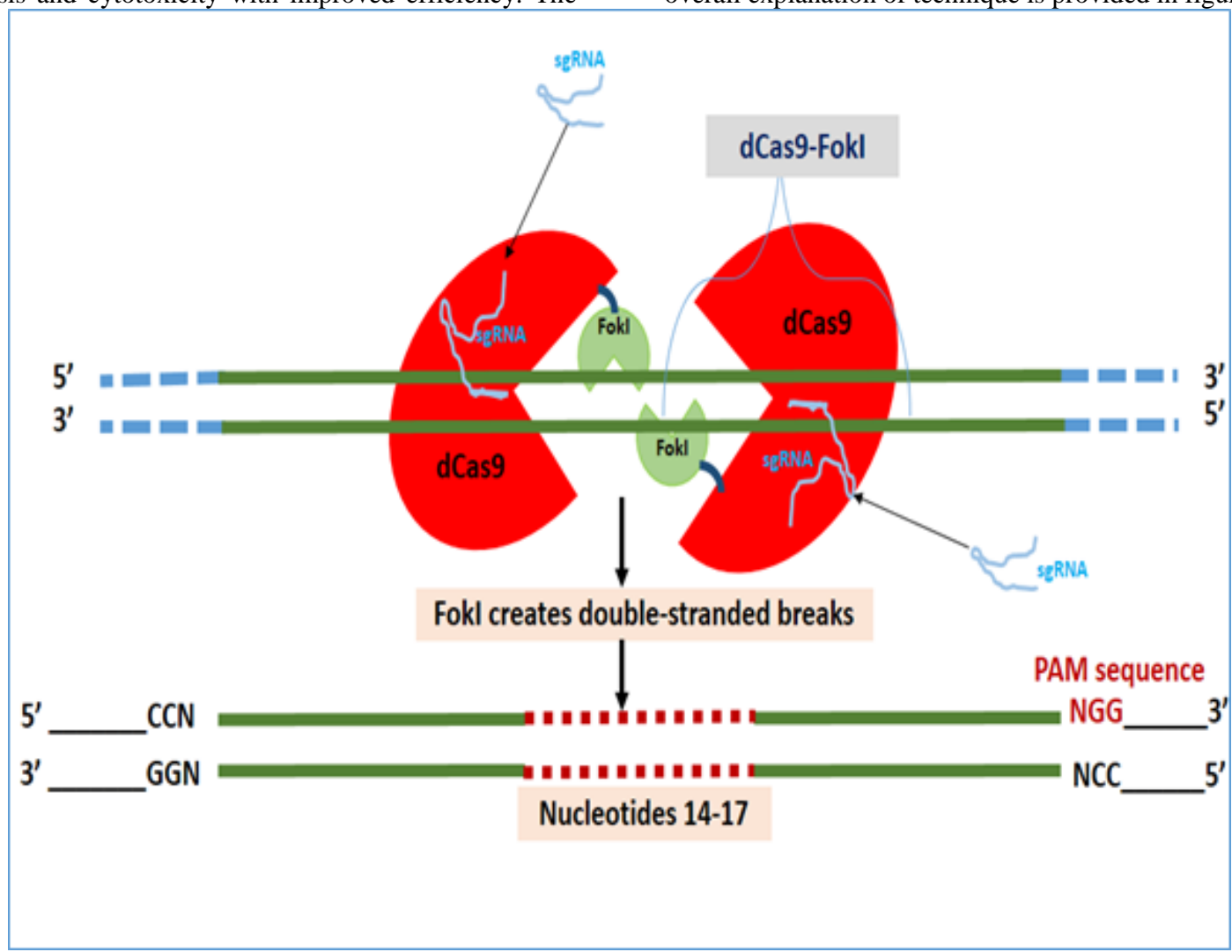

Figure-2: Tru-RFNs combining sRNA and Dimerization dependent RNA-guided FoKI-dCas 9 nucleases in creating highly specific double-stranded breaks in CRISPR/Cas 9 technology.

ii. Truncated guide RNAs (tru-gRNAs) CRISPR/Cas9 technology: CRISPR RNA guided nucleases (RGNs) are shorter version of nucleases with up to 20 complementary nucleotides with enhanced target specificity used in CRISPR/Cas technologies [20]. These truncations involve the 3" end of guide-RNA. These RNGs, being specific and shorter in length result in far less non-desirable mutagenesis and perform gene editing in a more efficient way. This version of CRISPR technology can be multiplexed easily, employed in regulatory transcriptional domains along with gRNA library preparation at genome-wide scale [21-22].

iii. Paired Cas9 nickase: Gopalappa et al have demonstrated that Cas9 paired nickases demonstrated higher efficiency than Cas9 nucleases [23]. However, Ren et al have shown less mutation rate with use of nickases but at the cost of low efficiency and thus considered the technique less compatible with high-throughput genome editing [24]. So what makes the use of nickases to have a better genome edited yield. In practical terms nickases have long overhangs instead of blunt ends which help appropriate insertion and match for the inserted segment thus limiting off-target effects [25].

iv. CRISPR/Cas9 with chemical treatment of sgRNA: DNA-free genome editing has been tried earlier with both TALENS and CRISPR, recent modifications of sgRNA by Hendel et al with certain chemicals including 2'-O-Methyl, 2'-O-Methyl + Phosphorothioates and 2'-O-Methyl + 3'-ThioPACE have been shown to be less toxic and more efficient [26]. Another very relevant study highlighted that both gRNAs and sgRNAs generate a potent interferon related immune response, which can be reduced by modifying 5'-Triphosphate group on removing phosphatases [27]. Similarly, Kelley et al have shown the versatile potential of chemically synthesized gRNA in terms of reduced off-target effects and possibility of high throughput CRISPR editing [28]. In simplistic terms chemical treatment of sgRNA/gRNA remains beneficial with minimal contrasting evidence.

v. CRISPR/Cpf1 System: Cpf-1 stands for "CRISPR associated Endonucleases in Prevotella and Francisella type-1". In comparison to its counterpart Cas protein, it is smaller with one RNA requirement and targets a T rich PAM site (TTTN) which is located distally to recognition site. Unlike the Cas9 induced blunt end cuts, it creates cuts in a zig zag manner along with requiring a small gRNA [29, 30]. Tsukamoto et al have observed reduced mutation rates and enhanced viability during manipulation of human hepatocytes using CRISPR/Cpf1 system [31]. Similarly, other authors have also mentioned advantages in terms of speed, efficiency, specificity and multiplexing option in possible real-time clinical deployment of genome editing [32]. 


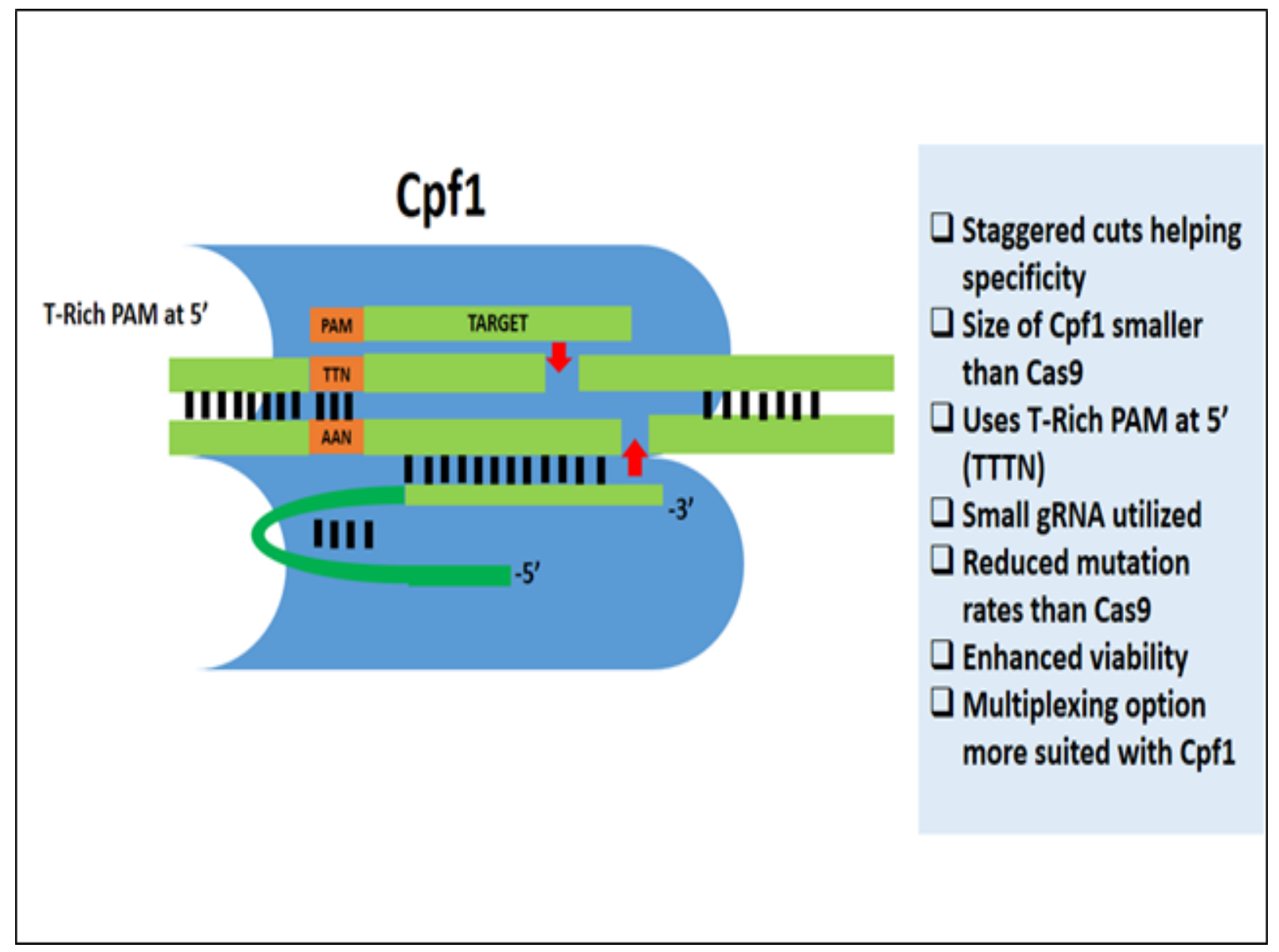

Figure-3: Cpf1 protein shown with PAM at 5'-end and genome edited gene product

vi. CRISPR/Cas6 with Csm / Cmr complex: From the study "The New Bacteriology" Hille et al have classified the type-III as A and B involving Csm and $\mathrm{Cmr}$, which are meant for both DNA and its transcript breakdown [33]. While the author could not find a biotechnological usage in literature, still it has been established that the two proteins have endoribonuclease activity to cleave down RNA rather than DNA $[34,35]$. The new age microbiology may find its possible use in defining antimicrobial resistance and diagnostic studies $[2,33,34]$.

The literature dealing with finding ways to increase the fidelity and efficiency of cleaving protein i.e., nucleases like various versions of Cas proteins or cpf 1 and possibly others are refining by every single day to improve the incoming new era clinically applicable gene therapy promise.

b. CRISPR payload delivery mechanisms: The payload including the specific Cas proteins, gRNA/sgRNA and other elements needs to be exactly delivered into cell and nucleus for accurate and efficient genome editing. This aspect is not only a biotechnological challenge but improvising the known obstacles is a well-recognized area of opportunity for optimized genome editing. Multiple modalities including direct, viral and non-viral modes are being experimented and the common ones are being discussed below:

i. Carrier free direct delivery of Cas9 RNPs: Pubmed survey over last 10 years identified 3 studies dealing with direct delivery Cas9 RNPs. Qiao et al have utilized Chitosan-coated red-fluorescent protein for delivery of both tagged Cas9 nuclease and donor DNAs with success [36]. Similar to above technique a study from Korea has developed direct delivery mode by developing fusion complex comprising Cas9 protein and low molecular weight protamine in conjunction with guide RNA with low immunogenicity and potential use for high-through put genome editing [37]. An in vitro study on zebrafish by Burger et al, Cas9 and related payload was directly moved into cell by using a soluble salt solution with efficient genome editing [38]. However, the studies need replication in realtime and a wider scale.

ii. Viral delivery mechanisms- Viral delivery modes of CRISPR Cas payload delivery were among the initial methods selected for use. The initial deployment was associated with multiple issues starting from carriage space, deranged immunity and prolong Cas expression duration thus compromising the role of these carriers as delivery mechanism [39]. However, these vectors are being modified in multiple ways resulting in slight improvements in terms of feasibility and efficiency [40]. Below is a brief about various virus related carriers used in delivering CRISPR/Cas machinery into cells and nuclei.

a) Adeno-associated viruses (AAVs): These viruses are nonenveloped single stranded DNA viruses which are considered to be least pathological in biological systems like human body [41]. AAVs surfaced as the pioneers in terms of viral vectors in gene therapy and preference overtime resulted due to decreased immunogenicity, low cytotoxicity, feasibility of usage and most importantly approval for clinical trials [42, 43]. Issues overtime encountered were related to decreased payload carriage capacity, being only suitable for loss of function mutations and decreased efficiency in wholesome. 
$[44,42]$ Provided improvised versions of AAVs entered the research market like dual and triple AAVs, modified mechanisms helping payload entry into cell like fragmentation, hybrid AAV vectors, overlapping transgenes still compromise the maximum capacity for allowing CRISPR/Cas machinery remain non-optimal and a major barrier for its futuristic transformation of gene therapy techniques [45-47].

b) Recombinant Adeno Associated Viruses (rAAV): Adenoviruses are double stranded, non-enveloped and nucleocapsid as an icosahedral structure. The characteristic which distinguish these viruses from AAVs is the inability to integrate inside the host cellular genome thus resulting in lower off-target effects [48]. Creating recombinant Adeno associated viruses (rAAVs) was a key step in improving viral delivery of gene therapy payloads into cells. Provided host acceptability, feasibility for transduction, approval for use in clinical trials and low cytotoxicity and low carriage capacity remained as a key hindrance in use with initial versions of these recombinant viruses which was later overcome by depleting viral genes like E2 and E4 and creating "Gutless Adenoviruses" or "Helper Dependent Adenoviruses" [49, 50]. Provided limitations rAAV induced gene transduction has been utilized in multiple studies with variable degree of success along with CRISPR/Cas and associated gene therapy techniques [42, 50, 51].

c) Lentivirus (LV) as delivery vehicle: Lentiviruses are retroviruses with single-stranded RNA which have been engineered as delivery vehicle for use in gene therapy [52, 42]. LV's ability to be tailored according to the incorporation needs by removing specific viral proteins and incorporating delivery payload and other structures makes it an attractive bioengineering tool for use in gene therapy [53]. Being depleted of most self-owned genes, the virus becomes least immunogenic and thus tolerated by host cells [53, 54]. However, being a retrovirus it can integrate into host genome to cause off-target mutagenesis (OTMs) [53]. To overcome these OTMs novel bioengineering techniques have been developed by creating silencing mutations in integrase enzyme genes to create "Non-integrating LVs" [55]. The limitations associated with use of Non-integrating LVs are logistical in terms of cost and availability to disallow its used in gene therapy but still it's being use for generating animal models for identifying diagnostics and therapeutic targets in cell lines $[56,57]$.

d) Delivery by artificial viruses: Recently, there is a surge in artificial viral vectors with variable and specific usage in literature. Li et al have described a "Multi-functional Nucleustargeting Core Shell Artificial Virus" (RRPHC) for delivering CRISPR payload delivery showing efficient in vivo silencing of MTH1 gene [58]. Similar efficiency for RRPHC in CRISPR payload delivery was also documented by others [59]. Zheng et al have added psuodo-rabies virus (PRV) clones into Bacterial Artificial Chromosomes (BAC), which were later utilized in efficient genome editing using CRISPR technology by incorporating fused Cas9 with cytosine deaminase [60]. Kong et al have developed biodegradable Peptidly Virus Like Particles ( $p$ VLP) which can carry the payload of CRISPR arsenal into cell in an efficient manner with better fidelity in terms genome editing [61]. MultiBac system, developed through a DNA recombination technique using Baculovirus apart from various other utilities have been found useful for transferring larger pay load through transfer plasmids in CRISPR/Cas9 system [62]. The above data with regards to artificial viral vectors indicates that many of the deficiencies and issues demonstrated by live viruses or recombinant viruses can be managed by artificial viral vectors. Though this bioengineering technique has just recently surfaced, but it is anticipated to take on gene therapy in some modes including CRISPR technologies. [60-62]

e) Bacteriophages: By definition a bacteriophage is a virus which infects a bacteria; however this potential of phage viruses are utilized in CRISPR technologies in multiple ways. These phage viruses have been bioengineered to create success stories in targeting antimicrobials resistance, vaccine delivery and gene therapy payload delivery vehicles [63]. Citorik et al have utilized customized RNA-guided nucleases i.e., gRNA + nuclease (RGNs) which were able to target specified DNA targets in host to disrupt genes associated with causing antibiotic resistance [64]. Bakhshinejad et al have termed the latest biotechnologically modified phages to be promising as gene delivery vehicles [65]. However, literature review also suggest lower efficiency in gene delivery via use of bacteriophages in comparison other viral and non-viral modes of gene carrying vectors [66].

iii. Non-viral delivery modes- Viral strategies, albeit showing successes still remain limited due to problems of immunogenicity, lesser carriage capacity and most importantly specificity [67, 42]. Alongside the biotechnological plight of some viral strategies, research remained useful to develop non-viral viral gene delivery vehicles specifically for delivering CRISPR payload into cell and nuclei. While so much data is available on non-viral methods of cellular entry of gene therapy apparatus only most discussed ones will be overviewed.

\section{a) Physical methods:}

1) Electroporation: This method utilizes an electrical pulse stimulation to induce pores on cell membrane for a short span a time which allows entry of CRISPR or other gene therapy related machinery to enter into cell and nuclei. Though looking crude still $\mathrm{Yu} \mathrm{J}$ have achieved successful results in knocking out malignant cells in malignant $\mathrm{B}$ cell using CRISPR/Cas9 technology [68]. Similarly, Bosch et al have used this technique with CRISPR/Cas9 for inducing tagging genes by fluorescent markers in cultured cells in animal models [69] .

2) Sonoporation: While the data for using this technique is limited for CRISPR technologies, still a study by Yoon et al utilized very high frequency ultrasound near cell membranes to deliver CRISPR/Cas payloads into cells and nucleus with precision [70]. In general this acoustic transfection mechanism of gene transfer modality has been used in many other ways for gene therapy $[71,72]$.

3) Magnetofection: This techniques employs a magnetic field by using magnetized nanoparticles for delivery of gene therapy machinery into cells. Hryhorowicz et al have deployed very small magnetic particle to transfer CRISPR/Cas constructs into cell for gene editing of fibroblasts in animal models [73].

4) Microinjection: This technique, though not specifically being utilized in CRISPR Cas technology still been utilized by some researchers [74]. 
b) Chemical methods: Chemical methods imply methodologies incorporating chemicals or synthetic structures which can carry gene therapy constructs into the cell. A description of important methods is as below:

Polymers: "Polymers" are generally made up of multiple identical subunits. They have multiple usages including delivery of CRISPR and other gene therapy payloads across the cellular membranes. Ryu et al have utilized Branchedpolyethyleneimine (PEI) polymers for CRISPR /Cas9 delivery with success [75]. Similarly, Zhang et al recently used a cationic polymer termed Polyethyleneimine with Betacyclodextrin to transfer Cas9 and associated constructs delivery into cell in an efficient manner [76].

Liposomes: These are small spherical structures resembling a sac and are composed of phospholipid membrane which have utilized for delivery of drug, vaccine and recently for delivering tools for gene therapy. These liposomes with cationic formulations have been demonstrated as a good carrier of CRISPR/Cas9 machinery for genome editing [77]. Similarly, Jubair et al have demonstrated pegylated liposome to be efficient CRISPR/Cas9 payload transporters across cell membranes for tumor treatment in animal models [78].

Peptides: Synthetic peptides which can translocate gene therapy constructs across cell membrane are usually termed as Cell Penetrating Peptides (CPP). These CPPs form complexes with Cas and gRNA to transport them into cells as demonstrated by Ramakirishna et al who were to efficiently disrupt CCR5 locus in various cancer cell lines with very few OTMs [79]. Similarly others have also been able to demonstrate the capability of CPPs in taxying CRISPR related payloads into cells [80]. Shen et al developed another novel mechanism utilizing amphipathic peptides (Endo-Porter) for delivering CRISPR gene editing machinery into cells. This technique is termed as "CRISPR-Delivery Particles" (CriPs) and the authors described it to have better efficiency, no detectable off-target effects and having therapeutic potential [81].

Nanoparticle: Nanoparticles (NPs) are small structures which apart from their uses in other bio techniques, are now being employed in various differing ways in relation to gene therapy. Mout et al have used NPs with modified arginine-gold assembled CRISPR tools including sgRNA and Cas9 for genome for delivery in targeted cells for genome editing with up to $30 \%$ efficiency [82]. Alsaiari et al developed a biocompatible nano framework of zeolite imidazole for efficient encapsulation of the tools for CRISPR genome editing. The specific advantages described with this technique included enhanced uploading, biocompatibility, escaping endosomal uptake and efficiency apart from common advantages of using chemical methodology [83]. "DNA Nanoclews" are small sewing thread like NPs which were used as a vehicle for CRISPR/Cas9 tool box delivery into cell. Salient advantages of this technique include enhanced efficiency and reduced immunogenicity [84]. Lipid like NPs have successfully utilized for CRISPR/Cas genome editing tools transfer via injections in animal models to reduce PCSK9 levels and viral loads of HBV DNA [85]. Magnetic NPs have also been utilized by creating a magnetic field allowing easy transfer of CRISPR Cas machinery into cell [86].

Cell-derived membrane vesicles (CMVs): CMVs have been utilized with delivery of siRNA and miNRA delivery into cells for gene therapy as demonstrated by Van Dommelen et al, but literature review does not provide its use with CRISPR/Cas9 methods [87, 88]. However, it will be an excellent idea to use this biologically compatible membrane for delivering CRISPR.

Other methods: Different variations from the above methods are also in vogue, which slightly differ due to differing combinations. Some of the examples are discussed. A Nanoblades delivery system was developed $\mathrm{Ma}$ et al for specifically targeting hematopoietic stem cells (HSCs) by CRISPR/Cas9 gene editing. This design was specific to HSC and resulted improved payload delivery, minimal off-target effects, reduced turnaround times, may provide an avenue for high-throughput genome editing approach with potential therapeutic benefits [89]. Carlson-Stevermer et al described the use of a RNA aptamer with streptavidin (S1mplex) which could join with CRISPR for optimized delivery of arsenal needed for gene editing with better precision, reducing turnaround times and cost with potential for future therapeutic delivery [90]. Another combination approach developed by Miller et al using "Zwitterionic Amino Lipids" (ZALs) for delivering of toolkit of CRISPR machinery into cell. This technique was able to deliver long RNAs with marked safety and futuristic therapeutic potential [91].

In general chemical methods have shown marked flexibility for bioengineering approaches, for disease-specific gene therapy payload delivery, feasibility and ease of transfer across cell membrane, minimal off-target mutagenesis and side effects, efficiency, reduced turnaround times, better management of carriage issues and most significantly being useful in highthroughput genome editing requirements. However, most of these techniques are required to be replicated in multi-centric trials, needing approval of regulatory authorities and translation into research and clinical practice. [71, 75, 83-85, 89-91]

A comparative generalization about various modes of CRISPR gene editing machinery transfer into cell is presented in table-1. 


\begin{tabular}{|c|c|c|c|c|}
\hline \multirow[t]{2}{*}{ Parameter } & \multirow{2}{*}{$\begin{array}{l}\text { Carrier free } \\
\text { direct delivery } \\
\text { of Cas } 9 \text { RNPs }\end{array}$} & \multirow{2}{*}{$\begin{array}{l}\text { Viral delivery } \\
\text { methods }\end{array}$} & \multicolumn{2}{|c|}{ Non-viral delivery modes } \\
\hline & & & Physical & Chemical \\
\hline \multicolumn{5}{|l|}{ Payload delivery } \\
\hline Vehicle selection/ need & - & $t+t+t^{45-47}$ & - & t+t+ \\
\hline Carriage capacity & $++^{36,37}$ & $+($ Up to $10-15 \mathrm{~Kb}$ ) $45-47$ & +t+ & $+++^{84,91,79,77}$ \\
\hline Carriage delivery & $++^{36,37}$ & $+(\text { Not predictable) })^{4547}$ & t+t & $+++++^{91,79,77}$ \\
\hline Bio-distribution & $+t^{37}$ & ++ (Variable) & t+t+ & $+++t^{83}$ \\
\hline \multicolumn{5}{|l|}{ Genome editing } \\
\hline Efficiency & $t t^{38}$ & ++ (Variable) & $+++^{70,72}$ & $+++++^{79,91,85}$ \\
\hline Fidelity & $+/-$ & ++ & t++ & $+++t^{91,83}$ \\
\hline Cytotoxicity & + & t+t & - & $+/ .84,81,91$ \\
\hline Immunogenicity & $t^{37}$ & t+t & + & $+/ .84,81,91$ \\
\hline Off-Target Mutagenesis (OTMs) & & $+t+t^{53}$ & - & $+/ .81,84,89$ \\
\hline High-throughput editing & $+t^{37}$ & ++ & t+t & t+t+ \\
\hline Regulatory body approvals & $\cdot$ & $+++t^{42,43}$ & - & $+1-$ \\
\hline Research use frequency & + & $+t+t^{42,43,56,57}$ & $t^{71,72}$ & $++^{85}$ \\
\hline Clinical use frequency & $+1-$ & $++t^{42,43,56}$ & $t^{71,72}$ & $++^{85}$ \\
\hline Therapeutic use & $+1-$ & $++(\text { LOF mutations) })^{44,42}$ & $+t^{69}$ & $++t^{84,85,89}$ \\
\hline Technique Modification Potential & t+ & + & ++ & $++++^{76,79,84,85,91}$ \\
\hline
\end{tabular}

Table-2: Generalized comparison between various delivery modes for CRISPR gene machinery

\section{c. gRNA free one-step CRISPR \& related technologies:}

i. CRISPR/Cas9-assisted gRNA-free-one-step (CAGO) techniques: This techniques suits well once face with CRISPR tolerant regions like mutations nearing 5'-end of protospacer and regions not containing PAM sequence (NGG). Furthermore this technology allows manipulation of large area genome editing with claimed efficiency of up to $75 \%$ [92].

ii. Oligonucleotide \& Polynucleotide strategies: These strategies use oligomers or short polymers which can introduce DNA modifications in a specific targeted DNA sequence like homologous exchange mechanism. While the detailed insight about molecular understanding of this process is still being deciphered, it seems that the methodology use the inherent cell's DNA repair system and exchange the non-desirable fragment with foreign DNA segment [93]. This methodology could incorporate non-homologous repair end joining or HomologyDependent Repair (HDR) in combination [88, 93]. Currently this technique is rapidly expanding with various new biotechnological innovations and tailoring it to research clinical and research use. Various available platforms for such techniques from single stranded oligonucleotide to Triple Forming Oligonucleotides (TFOs). Attempts have been made for direct delivery of exogenous Nucleic Acids (single or double-stranded DNA) with quite a success [88]. "Single Fragment Homologous Replacement (SFHR)", though not a new addition, is a method where a where a very short DNA segment of size $1 \mathrm{~Kb}$ replaces a homologous region on host DNA. The technology being miniaturized avoids regulatory sequences like enhancers and suppressors and provides utmost fidelity in gene replacement along with an avenue for multiplexing [94, 95]. "Triplex-forming Oligonucleotides (TFO)" is showing much improvement in terms of biotechnological developments like miniaturization and multiplexed platforms. [96] However, TFO based technologies have shown practical issues in terms of limitations of use to only homo-purine sequences. They were not able to recognize pyrimidines as they possess only one-hydrogen acceptor in major DNA groove [97]. Further innovations to overcome this issue resulted in development of Peptide Nucleic Acids (PNAs) supplanted by nucleobases which could overcome this problem and were able to address this issue [97]. Further improvements on PNA strategies for gene therapy suggested this technique to be more promising and workable [98].

CAGO, PNA and TFO are newer in market with limitations in research and quality control. Though promising in multiple ways including feasibility, multiplexing and less cytotoxic still the data and experiments need to be replicated in further experimental set ups.

Emerging biotechnological innovations in gene therapy- We have discussed the various specific aspects of technologies close to CRISPR/Cas. However, the literature review alongside describes some other biotechnological innovations which in time may evolve and used with gene therapy. First we will make a mention of some new and differential technologies related to CRISPR/Cas system followed by some completely versatile novel approaches in gene therapy. Gao et al have used a protein "Argonaute" from a bacterium "Natronobacterium 
gregoryi”, called as $\mathrm{NgAgo}$ for genome editing, which don not need a PAM recognition sequence or an RNA. The system is termed as "NgAgo-gDNA system" having a single-stranded DNA with 24 nucleotides which remains very efficient for genome editing in gene areas without a PAM-sequence and GC region editing with very low non-desirable mutations [99]. However, some suthors have described some issues with the technique, so there is need to be replicate this system in other setups [100,101]. Recently, another technique has been developed which uses "Artificial Restirction DNA Cutter" (ARCUT), which incorporates a pseudo-complementary Peptide Nucelic Acids (pcPNA) with molecular scissors as Ce (IV) / EDTA structure with capability to induce site specific double stranded DNA cleavage [102]. This technique obviates the need of restriction enzymes and will pave way for chemical and protein base site-specific genome editing.

The next-stage in DNA manipulation and bioengineering will be the use of "synthetic biology" which will probably change the landscape of current gene modifying technologies [103]. Though ethical and technical issues will be required to address to reach clinical usage but future horizons in biotechnology seem to move towards "synthesis" rather than "editing", where the former may be partially available in various formats.

\section{Conclusion}

The development of CRISPR/Cas technologies for gene editing have geared up for becoming a real physician tool for providing panacea to patients. Though various search engines with Key words "CRISPR" just provide an overwhelming number of data, still the technology seem to be suboptimal at this time which is depicted by numerous experimental approaches adopted to modify gene editing tools including nucleases and delivery pathways. While the recent data in many ways seem adequate but shortcomings in terms of plethora of off-target effects, reduced efficacies and less desirable delivery issues, which create its ultimate limitation for complete clinical application. This review attempted to integrate various nucleases, nuclease-free approaches to genome editing along with discussing the modes available for gene editing payload delivery into cell nuclei. There seem to be shift from conventional CRISPR/Cas9 system to non-Cas nucleases or nuclease independent technologies along with more reliance on non-viral chemical modes of delivering gen editing constructs. An unmet need is also felt to consolidate various methodologies by a dedicated regulatory body to avoid both short-term and long-term technical and ethical issues.

\section{Declarations-}

\section{Consent for publication:}

Not applicable (No individual data was presented)

\section{Competing interests-}

The author has no competing interests to declare.

\section{Data funding-}

There are no funding sources to disclose.

\section{References}

1. Mojica FJ, Díez-Villaseñor C, García-Martínez J, Soria E. (2005) Intervening sequences of regularly spaced prokaryotic repeats derive from foreign genetic elements. J Mol Evol. 60(2):174-182.

2. Koonin EV, Makarova KS, Zhang F. (2017) Diversity, classification and evolution of CRISPR-Cas systems. Curr Opin Microbiol. 37:67-78.

3. Jinek M, Chylinski K, Fonfara I, Hauer M, Doudna JA, Charpentier E. (2012) A programmable dual-RNA-guided DNA endonuclease in adaptive bacterial immunity. Science. 337(6096):816-821.

4. Urnov FD, Rebar EJ, Holmes MC, Zhang HS, Gregory PD. (2010) Genome editing with engineered zinc finger nucleases. Nat Rev Genet. 11(9):636-646

5. Bogdanove AJ, Voytas DF. (2011) TAL effectors: customizable proteins for DNA targeting. Science. 333(6051):1843-1846. doi: 10.1126/science.1204094.

6. Doudna JA, Charpentier E. (2014) Genome editing. The new frontier of genome engineering with CRISPR-Cas9. Science. 346(6213):1258096. doi: 10.1126/science.1258096.

7. Kimberland ML, Hou W, Alfonso-Pecchio A, Wilson S, Rao Y, Zhang S, Lu Q. (2018) Strategies for controlling CRISPR/Cas9 off-target effects and biological variations in mammalian genome editing experiments. J Biotechnol. 284:91-101. doi: 10.1016/j.jbiotec.2018.08.007.

8. Chew WL, Tabebordbar M, Cheng JK, Mali P, Wu EY, et al. (2016) A multifunctional AAV-CRISPR-Cas9 and its host response. Nat Methods. 13(10):868-874. doi: 10.1038/nmeth.3993.

9. Wang W, Ye C, Liu J, Zhang D, Kimata JT, Zhou P. (2014) CCR5 gene disruption via lentiviral vectors expressing Cas9 and single guided RNA renders cells resistant to HIV-1 infection. PLoS One. 9(12):e115987. doi: 10.1371/journal.pone.0115987. eCollection 2014.

10. Wang G, Zhao N, Berkhout B, Das AT. (2016) A Combinatorial CRISPR-Cas9 Attack on HIV-1 DNA Extinguishes All Infectious Provirus in Infected $\mathrm{T}$ Cell Cultures. Cell Rep. 17(11):2819-2826. doi: 10.1016/j.celrep.2016.11.057.

11. Zhao D, Feng X, Zhu X, Wu T, Zhang X, Bi C. (2017) CRISPR/Cas9-assisted gRNA-free one-step genome editing with no sequence limitations and improved targeting efficiency. Sci Rep. 7(1):16624. doi: 10.1038/s41598-017-16998-8.

12. Adli M. (2018) The CRISPR tool kit for genome editing and beyond. Nat Commun. 9(1):1911. doi: 10.1038/s41467-01804252-2.

13. Barrangou R. (2015) The roles of CRISPR-Cas systems in adaptive immunity and beyond. Curr Opin Immunol. 32:36-41. doi: 10.1016/j.coi.2014.12.008.

14. Eid A, Mahfouz MM. (2016) Genome editing: the road of CRISPR/Cas9 from bench to clinic. Exp Mol Med. 48(10):e265. doi: 10.1038/emm.2016.111.

15. Regalado, Antonio (25 November 2018). "Exclusive: Chinese scientists are creating CRISPR babies - A daring effort is under way to create the first children whose DNA has been tailored using gene editing". MIT Technology Review. Retrieved 26 November 2018. (Cross-reference)

16. Lin Y, Cradick TJ, Brown MT, Deshmukh H, Ranjan P, Sarode N, et al. (2014) CRISPR/Cas9 systems have off-target activity with insertions or deletions between target DNA and guide RNA sequences. Nucleic Acids Res. 42(11):7473-7485. doi: 10.1093/nar/gku402.

17. Jinek M, Chylinski K, Fonfara I, Hauer M, Doudna JA, Charpentier E. (2012) A programmable dual-RNA-guided DNA endonuclease in adaptive bacterial immunity. Science. 337(6096):816-821. doi: $10.1126 /$ science.1225829.

18. Mehta A, Merkel OM. (2019) Immunogenicity of Cas9 Protein. J Pharm Sci. pii: S0022-3549(19)30637. doi: 10.1016/j.xphs.2019.10.003.

19. Wyvekens N, Topkar VV, Khayter C, Joung JK, Tsai SQ. (2015) Dimeric CRISPR RNA-Guided FokI-dCas9 Nucleases Directed by Truncated gRNAs for Highly Specific Genome Editing. Hum Gene Ther. 26(7):425-431. doi: 10.1089/hum.2015.084. 
20. An L, Hu Y, Chang S, Zhu X, Ling P, Zhang F, et al. (2016) Efficient generation of FVII gene knockout mice using CRISPR/Cas9 nuclease and truncated guided RNAs. Sci Rep. 6:25199. doi: 10.1038/srep25199.

21. Cong L, Ran FA, Cox D, Lin S, Barretto R, Habib N, et al. (2013) Multiplex genome engineering using CRISPR/Cas systems. Science. 339(6121):819-823.

22. Shalem O, Sanjana NE, Hartenian E, Shi X, Scott DA, Mikkelson T, et al. (2014) Genome-scale CRISPR-Cas9 knockout screening in human cells. Science. 343(6166):84-87. doi: $10.1126 /$ science. 1247005 .

23. Gopalappa R, Suresh B, Ramakrishna S, Kim HH. (2018) Paired D10A Cas9 nickases are sometimes more efficient than individual nucleases for gene disruption. Nucleic Acids Res. 46(12):e71. doi: 10.1093/nar/gky222.

24. Ren X, Yang Z, Mao D, Chang Z, Qiao HH, Wang X,et al. (2014) Performance of the Cas9 nickase system in Drosophila melanogaster. G3 (Bethesda). 4(10):1955-1962. doi: 10.1534/g3.114.013821.

25. Mali P, Aach J, Stranges PB, Esvelt KM, Moosburner M, Kosuri $\mathrm{S}$, et al. (2013) CAS9 transcriptional activators for target specificity screening and paired nickases for cooperative genome engineering. Nat Biotechnol. 31(9):833-838. doi: 10.1038/nbt. 2675 .

26. Hendel A, Bak RO, Clark JT, Kennedy AB, Ryan DE, Roy S, et al. (2015) Chemically modified guide RNAs enhance CRISPRCas genome editing in human primary cells. Nat Biotechnol. 33(9):985-989. doi: 10.1038/nbt.3290.

27. Schubert MS, Cedrone E, Neun B, Behlke MA, Dobrovolskaia MA. (2018) Chemical Modification of CRISPR gRNAs Eliminate type I Interferon Responses in Human Peripheral Blood Mononuclear Cells. J Cytokine Biol. 3(1). pii: 121. doi: 10.4172/2576-3881.1000121.

28. Kelley ML, Strezoska Ž, He K, Vermeulen A, Smith Av. (2016) Versatility of chemically synthesized guide RNAs for CRISPRCas9 genome editing. J Biotechnol. 233:74-83. doi: 10.1016/j.jbiotec.2016.06.011.

29. Safari F, Zare K, Negahdaripour M, Barekati-Mowahed M, Ghasemi Y. (2019) CRISPR Cpf1 proteins: structure, function and implications for genome editing. Cell Biosci. 9:36. doi: 10.1186/s13578-019-0298-7.

30. Bayat H, Modarressi MH, Rahimpour A. (2018) The Conspicuity of CRISPR-Cpf1 System as a Significant Breakthrough in Genome Editing. Curr Microbiol. 75(1):107-115. doi: 10.1007/s00284-017-1406-8.

31. Tsukamoto T, Sakai E, Iizuka S, Taracena-Gándara M, Sakurai F, Mizuguchi H. (2018) Generation of the Adenovirus VectorMediated CRISPR/Cpf1 System and the Application for Primary Human Hepatocytes Prepared from Humanized Mice with Chimeric Liver. Biol Pharm Bull. 41(7):1089-1095. doi: 10.1248/bpb.b18-00222.

32. Verwaal R, Buiting-Wiessenhaan N, Dalhuijsen S, Roubos JA. (2018) CRISPR/Cpf1 enables fast and simple genome editing of Saccharomyces cerevisiae. Yeast. 35(2):201-211. doi: 10.1002/yea.3278.

33. Hille F, Charpentier E. (2016) CRISPR-Cas: biology, mechanisms and relevance. Philos Trans R Soc Lond B Biol Sci. 371(1707). pii: 20150496. doi: 10.1098/rstb.2015.0496.

34. Staals RH, Zhu Y, Taylor DW, Kornfeld JE, Sharma K, Barendregt A, et al. (2014) RNA targeting by the type III-A CRISPR-Cas Csm complex of Thermus thermophilus. Mol Cell. 56(4):518-530. doi: 10.1016/j.molcel.2014.10.005.

35. Guo M, Zhang K, Zhu Y, Pintilie GD, Guan X, Li S, et al. (2019) Coupling of ssRNA cleavage with DNase activity in type
III-A CRISPR-Csm revealed by cryo-EM and biochemistry. Cell Res. 29(4):305-312. doi: 10.1038/s41422-019-0151-x.

36. Qiao J, Sun W, Lin S, Jin R, Ma L, Liu Y. (2019) Cytosolic delivery of CRISPR/Cas9 ribonucleoproteins for genome editing using chitosan-coated red fluorescent protein. Chem Commun (Camb). 55(32):4707-4710. doi: 10.1039/c9cc00010k.

37. Kim SM, Shin SC, Kim EE, Kim SH, Park K, Oh SJ, Jang M. (2018) Simple in Vivo Gene Editing via Direct Self-Assembly of Cas9 Ribonucleoprotein Complexes for Cancer Treatment. ACS Nano. 12(8):7750-7760. doi: 10.1021/acsnano.8b01670.

38. Burger A, Lindsay H, Felker A, Hess C, Anders C, Chiavacci E, et al. (2016) Maximizing mutagenesis with solubilized CRISPRCas9 ribonucleoprotein complexes. Development. 143(11):20252037. doi: 10.1242/dev.134809.

39. Wilbie D, Walther J, Mastrobattista E. (2019) Delivery Aspects of CRISPR/Cas for in Vivo Genome Editing. Acc Chem Res. 52(6):1555-1564. doi: 10.1021/acs.accounts.9b00106.

40. Chandrasekaran AP, Song M, Kim KS, Ramakrishna S. (2018) Different Methods of Delivering CRISPR/Cas9 Into Cells. Prog Mol Biol Transl Sci. 159:157-176. doi: 10.1016/bs.pmbts.2018.05.001.

41. Hastie E, Samulski RJ. (2015) Adeno-associated virus at 50: a golden anniversary of discovery, research, and gene therapy success--a personal perspective. Hum Gene Ther. 26(5):257-265. doi: 10.1089/hum.2015.025.

42. Xu CL, Ruan MZC, Mahajan VB, Tsang SH. (2019) Viral Delivery Systems for CRISPR. Viruses. 11(1). pii: E28. doi: 10.3390/v11010028.

43. Lau CH, Suh Y. (2017) In vivo genome editing in animals using AAV-CRISPR system: applications to translational research of human disease. Flo00Res. 6:2153. doi: 10.12688/f1000research.11243.1.

44. Peng R, Lin G, Li J. (2016) Potential pitfalls of CRISPR/Cas9mediated genome editing. FEBS J. 283(7):1218-1231. doi: 10.1111/febs. 13586

45. Maddalena A, Tornabene P, Tiberi P, Minopoli R, Manfredi A, Mutarelli M, et al. (2018) Triple Vectors Expand AAV Transfer Capacity in the Retina. Mol Ther. 26(2):524-541. doi: 10.1016/j.ymthe.2017.11.019.

46. Kodippili K, Hakim CH, Pan X, Yang HT, Yue Y, Zhang Y, et al. (2018) Dual AAV Gene Therapy for Duchenne Muscular Dystrophy with a 7-kb Mini-Dystrophin Gene in the Canine Model. Hum Gene Ther. 29(3):299-311. doi: 10.1089/hum.2017.095.

47. Ghosh A, Yue Y, Lai Y, Duan D. (2008) A hybrid vector system expands adeno-associated viral vector packaging capacity in a transgene-independent manner. Mol Ther. 16(1):124-130.

48. Crenshaw BJ, Jones LB, Bell CR, Kumar S, Matthews QL. (2019) Perspective on Adenoviruses: Epidemiology, Pathogenicity, and Gene Therapy. Biomedicines. 7(3). pii: E61. doi: 10.3390/biomedicines7030061.

49. Zhou ZP, Yang LL, Cao H, Chen ZR, Zhang Y, Wen XY, Hu J. (2019) In Vitro Validation of a CRISPR-Mediated CFTR Correction Strategy for Preclinical Translation in Pigs. Hum Gene Ther. 30(9):1101-1116. doi: 10.1089/hum.2019.074.

50. Li C, Ding L, Sun CW, Wu LC, Zhou D, Pawlik KM, et al. (2016) Novel HDAd/EBV Reprogramming Vector and Highly Efficient Ad/CRISPR-Cas Sickle Cell Disease Gene Correction. Sci Rep. 6:30422. doi: 10.1038/srep30422.

51. Yoon Y, Wang D, Tai PWL, Riley J, Gao G, Rivera-Pérez JA. (2018) Streamlined ex vivo and in vivo genome editing in mouse embryos using recombinant adeno-associated viruses. Nat Commun. 9(1):412. doi: 10.1038/s41467-017-02706-7. 
52. Zhao Y, Stepto H, Schneider CK. (2017) Development of the First World Health Organization Lentiviral Vector Standard: Toward the Production Control and Standardization of Lentivirus-Based Gene Therapy Products. Hum Gene Ther Methods. 28(4):205-214. doi: 10.1089/hgtb.2017.078.

53. Pauwels K, Gijsbers R, Toelen J, Schambach A, Willard-Gallo $\mathrm{K}$, et al. (2009) State-of-the-art lentiviral vectors for research use: risk assessment and biosafety recommendations. Curr Gene Ther. 9(6):459-474.

54. Gándara C, Affleck V, Stoll EA. (2018) Manufacture of ThirdGeneration Lentivirus for Preclinical Use, with Process Development Considerations for Translation to Good Manufacturing Practice. Hum Gene Ther Methods. 29(1):1-15. doi: 10.1089/hgtb.2017.098.

55. Hu J, Schokrpur S, Archang M, Hermann K, Sharrow AC, Khanna P, et al. (2018) A Non-integrating Lentiviral Approach Overcomes Cas9-Induced Immune Rejection to Establish an Immunocompetent Metastatic Renal Cancer Model. Mol Ther Methods Clin Dev. 9:203-210. doi: 10.1016/j.omtm.2018.02.009.

56. Huo W, Zhao G, Yin J, Ouyang X, Wang Y, Yang C, et al. (2017) Lentiviral CRISPR/Cas9 vector mediated miR-21 gene editing inhibits the epithelial to mesenchymal transition in ovarian cancer cells. $J$ Cancer. 8(1):57-64. doi: 10.7150/jca.16723. eCollection 2017.

57. Zhao G, Wang Q, Gu Q, Qiang W, Wei JJ, Dong P, et al. (2017) Lentiviral CRISPR/Cas9 nickase vector mediated BIRC5 editing inhibits epithelial to mesenchymal transition in ovarian cancer cells. Oncotarget. 8(55):94666-94680. doi: 10.18632/oncotarget.21863.

58. Li L, Song L, Liu X, Yang X, Li X, He T, et al. (2017) Artificial Virus Delivers CRISPR-Cas9 System for Genome Editing of Cells in Mice. ACS Nano. 11(1):95-111. doi: 10.1021/acsnano.6b04261.

59. Yang S, Wu Q, Wei Y, Gong C. (2019) CRISPR-Cas9 Delivery by Artificial Virus (RRPHC). Methods Mol Biol. 1961:81-91. doi: 10.1007/978-1-4939-9170-9_6.

60. Zheng K, Jiang FF, Su L, Wang X, Chen YX, Chen HC, Liu ZF. (2019) Highly Efficient Base Editing in Viral Genome Based on Bacterial Artificial Chromosome Using a Cas9-Cytidine Deaminase Fused Protein. Virol Sin. doi: 10.1007/s12250-01900175-4.

61. Kong J, Wang Y, Zhang J, Qi W, Su R, He Z. (2018) Rationally Designed Peptidyl Virus-Like Particles Enable Targeted Delivery of Genetic Cargo. Angew Chem Int Ed Engl. 57(43):14032-14036. doi: 10.1002/anie.201805868.

62. Pelosse M, Crocker H, Gorda B, Lemaire P, Rauch J, Berger I. (2017) MultiBac: from protein complex structures to synthetic viral nanosystems. BMC Biol. 15(1):99. doi: 10.1186/s12915017-0447-6.

63. Chen Y, Batra H, Dong J, Chen C, Rao VB, Tao P. (2019) Genetic Engineering of Bacteriophages Against Infectious Diseases. Front Microbiol. 10:954. doi: 10.3389/fmicb.2019.00954.

64. Citorik RJ, Mimee M, Lu TK. (2014) Sequence-specific antimicrobials using efficiently delivered RNA-guided nucleases. Nat Biotechnol. 32(11):1141-5. doi: 10.1038/nbt.3011.

65. Bakhshinejad B, Sadeghizadeh M. (2014) Bacteriophages as vehicles for gene delivery into mammalian cells: prospects and problems. Expert Opin Drug Deliv. 11(10):1561-74. doi: 10.1517/17425247.2014.927437.

66. Hosseinidoust Z. (2017) Phage-Mediated Gene Therapy. Curr Gene Ther. 17(2):120-126. doi: $10.2174 / 1566523217666170510151940$.
67. Wang K, Huang Q, Qiu F, Sui M. (2015) Non-viral Delivery Systems for the Application in 553 Cancer Gene Therapy. Curr Med Chem. 22(35):4118-4136.

68. Yu J. (2020) Electroporation of CRISPR-Cas9 into Malignant B Cells for Loss-of-Function Studies of Target Gene Via Knockout. Methods Mol Biol. 2050:85-90. doi: 10.1007/978-14939-9740-4_9.

69. Bosch JA, Knight S, Kanca O, Zirin J, Yang-Zhou D, Hu Y, et al. (2020) Use of the CRISPR-Cas9 System in Drosophila Cultured Cells to Introduce Fluorescent Tags into Endogenous Genes. Curr Protoc Mol Biol. 130(1):e112. doi: 10.1002/cpmb.112.

70. Yoon S, Wang P, Peng Q, Wang Y, Shung KK. (2017) Acoustictransfection for genomic manipulation of single-cells using high frequency ultrasound. Sci Rep. 7(1):5275. doi: 10.1038/s41598017-05722-1.

71. Rinaldi L, Folliero V, Palomba L, Zannella C, Isticato R, Di Francia R, et al. (2018) Sonoporation by microbubbles as gene therapy approach against liver cancer. Oncotarget. 9(63):3218232190. doi: 10.18632/oncotarget. 25875.

72. Mignet N, Marie C, Delalande A, Manta S, Bureau MF, Renault G, et al. (2019) Microbubbles for Nucleic Acid Delivery in Liver Using Mild Sonoporation. Methods Mol Biol. 1943:377-387. doi: 10.1007/978-1-4939-9092-4_25.

73. Hryhorowicz M, Grześkowiak B, Mazurkiewicz N, Śledziński P, Lipiński D, Słomski R. (2019) Improved Delivery of CRISPR/Cas9 System Using Magnetic Nanoparticles into Porcine Fibroblast. Mol Biotechnol. 61(3):173-180. doi: 10.1007/s12033-018-0145-9.

74. Hamann A, Nguyen A, Pannier AK. (2019) Nucleic acid delivery to mesenchymal stem cells: a review of nonviral methods and applications. $J$ Biol Eng. 13:7. doi: 10.1186/s13036-019-0140-0. eCollection 2019.

75. Ryu N, Kim MA, Park D, Lee B, Kim YR, Kim KH, et al. (2018) Effective PEI-mediated delivery of CRISPR-Cas9 complex for targeted gene therapy. Nanomedicine. 14(7):20952102. doi: 10.1016/j.nano.2018.06.009.

76. Zhang Z, Wan T, Chen Y, Chen Y, Sun H, Cao T, et al. (2019) Cationic Polymer-Mediated CRISPR/Cas9 Plasmid Delivery for Genome Editing. Macromol Rapid Commun. 40(5):e1800068. doi: 10.1002/marc.201800068.

77. Zhen S, Li X. (2019) Liposomal delivery of CRISPR/Cas9. Cancer Gene Ther. doi: 10.1038/s41417-019-0141-7.

78. Jubair L, Fallaha S, McMillan NAJ. (2019) Systemic Delivery of CRISPR/Cas9 Targeting HPV Oncogenes Is Effective at Eliminating Established Tumors. Mol Ther. 27(12):2091-2099. doi: 10.1016/j.ymthe.2019.08.012.

79. Ramakrishna S, Kwaku Dad AB, Beloor J, Gopalappa R, Lee SK, Kim H. (2014) Gene disruption by cell-penetrating peptidemediated delivery of Cas9 protein and guide RNA. Genome Res. 24(6):1020-1027. doi: 10.1101/gr.171264.113.

80. Suresh B, Ramakrishna S, Kim H. (2017) Cell-Penetrating Peptide-Mediated Delivery of Cas9 Protein and Guide RNA for Genome Editing. Methods Mol Biol. 1507:81-94.

81. Shen Y, Cohen JL, Nicoloro SM, Kelly M, Yenilmez B, Henriques F, et al. (2018) CRISPR-delivery particles targeting nuclear receptor-interacting protein 1 (Nrip1) in adipose cells to enhance energy expenditure. J Biol Chem. 293(44):17291-17305.

82. Mout R, Ray M, Yesilbag Tonga G, Lee YW, Tay T, Sasaki K, Rotello VM. (2017) Direct Cytosolic Delivery of CRISPR/Cas9Ribonucleoprotein for Efficient Gene Editing. ACS Nano. 11(3):2452-2458. doi: 10.1021/acsnano.6b07600.

83. Alsaiari SK, Patil S, Alyami M, Alamoudi KO, Aleisa FA, Merzaban JS,et al. (2018) Endosomal Escape and Delivery of 
CRISPR/Cas9 Genome Editing Machinery Enabled by Nanoscale Zeolitic Imidazolate Framework. J Am Chem Soc. 140(1):143-146. doi: 10.1021/jacs.7b11754.

84. Sun W, Ji W, Hall JM, Hu Q, Wang C, Beisel CL, Gu Z. (2015) Self-assembled DNA nanoclews for the efficient delivery of CRISPR-Cas9 for genome editing. Angew Chem Int Ed Engl. 54(41):12029-12033. doi: 10.1002/anie.201506030.

85. Jiang C, Mei M, Li B, Zhu X, Zu W, Tian Y, et al. (2017) A nonviral CRISPR/Cas9 delivery system for therapeutically targeting HBV DNA and pcsk9 in vivo. Cell Res. 27(3):440-443. doi: 10.1038/cr.2017.16.

86. Hryhorowicz M, Grześkowiak B, Mazurkiewicz N, Śledziński P, Lipiński D, Słomski R. (2019) Improved Delivery of CRISPR/Cas9 System Using Magnetic Nanoparticles into Porcine Fibroblast. Mol Biotechnol. 61(3):173-180. doi: 10.1007/s12033-018-0145-9.

87. van Dommelen SM, Vader P, Lakhal S, Kooijmans SA, van Solinge WW, et al. (2012) Microvesicles and exosomes: opportunities for cell-derived membrane vesicles in drug delivery. $J$ Control Release. 161(2):635-644. doi: 10.1016/j.jconrel.2011.11.021.

88. Piotrowski-Daspit AS, Glaze PM, Saltzman WM. (2018) Debugging the genetic code: non-viral in vivo delivery of therapeutic genome editing technologies. Curr Opin Biomed Eng. 7:24-32. doi: 10.1016/j.cobme.2018.08.002.

89. Ma Y, Han X, Quintana Bustamante O, Bessa de Castro R, Zhang K, Zhang P, et al. (2017) Highly efficient genome editing of human hematopoietic stem cells via a nano-silicon-blade delivery approach. Integr Biol (Camb). 9(6):548-554. doi: 10.1039/c7ib00060j.

90. Carlson-Stevermer J, Abdeen AA, Kohlenberg L, Goedland M, Molugu K, Lou M, Saha K. (2017) Assembly of CRISPR ribonucleoproteins with biotinylated oligonucleotides via an RNA aptamer for precise gene editing. Nat Commun. 8(1):1711. doi: 10.1038/s41467-017-01875-9.

91. Miller JB, Zhang S, Kos P, Xiong H, Zhou K, Perelman SS, Zhu H, Siegwart DJ. (2017) Non-Viral CRISPR/Cas Gene Editing In Vitro and In Vivo Enabled by Synthetic Nanoparticle CoDelivery of Cas9 mRNA and sgRNA. Angew Chem Int Ed Engl. 56(4):1059-1063. doi: 10.1002/anie.201610209.

92. Zhao D, Feng X, Zhu X, Wu T, Zhang X, Bi C. (2017) CRISPR/Cas9-assisted gRNA-free one-step genome editing with no sequence limitations and improved targeting efficiency. Sci Rep. 7(1):16624. doi: 10.1038/s41598-017-16998-8.

93. Sargent RG, Kim S, Gruenert DC. (2011) Oligo/polynucleotidebased gene modification: strategies and therapeutic potential. Oligonucleotides. 21(2):55-75. doi: 10.1089/oli.2010.0273.
94. Luchetti A, Malgieri A, Sangiuolo F. (2014) Small Fragment Homologous Replacement (SFHR): sequence-specific modification of genomic DNA in eukaryotic cells by small DNA fragments. Methods Mol Biol. 1114:85-101. doi: 10.1007/978-162703-761-7_6.

95. Pierandrei S, Luchetti A, Sanchez M, Novelli G, Sangiuolo F, Lucarelli M. (2016) The Gene Targeting Approach of Small Fragment Homologous Replacement (SFHR) Alters the Expression Patterns of DNA Repair and Cell Cycle Control Genes. Mol Ther Nucleic Acids. 5:e304. doi: 10.1038/mtna.2016.2.

96. Petralia S, Forte G, Zimbone M, Conoci S. (2019) The cooperative interaction of triplex forming oligonucleotides on DNA-triplex formation at electrode surface: Molecular dynamics studies and experimental evidences. Colloids Surf $B$ Biointerfaces. 110648. doi: 10.1016/j.colsurfb.2019.110648.

97. Kumpina I, Brodyagin N, MacKay JA, Kennedy SD, Katkevics M, Rozners E. (2019) Synthesis and RNA-Binding Properties of Extended Nucleobases for Triplex-Forming Peptide Nucleic Acids. J Org Chem. 84(21):13276-13298. doi: 10.1021/acs.joc.9b01133.

98. Zhang L, Linden G, Vázquez O. (2019) In search of visible-light photoresponsive peptide nucleic acids (PNAs) for reversible control of DNA hybridization. Beilstein J Org Chem. 15:25002508. doi: 10.3762/bjoc.15.243. eCollection 2019.

99. Gao F, Shen XZ, Jiang F, Wu Y, Han C. (2016) DNA-guided genome editing using the Natronobacterium gregoryi Argonaute. Nat Biotechnol. 34(7):768-773. doi: 10.1038/nbt.3547.

100. Nakade S, Yamamoto T, Sakuma T. (2017) Cas9, Cpf1 and C2c1/2/3-What's next? Bioengineered. 8(3):265-273. doi: 10.1080/21655979.2017.1282018.

101. Cyranoski D. (2016) Replications, ridicule and a recluse: the controversy over NgAgo gene-editing intensifies. Nature. 536(7615):136-137. doi: 10.1038/536136a.

102. Komiyama M. (2013) Cut-and-Paste of DNA Using an Artificial Restriction DNA Cutter. Int J Mol Sci. 14(2):3343-3357. doi: 10.3390/ijms14023343.

103. Hong SH. (2019) Cell-Free Synthetic Biology: Synthetic Biology Meets Cell-Free Protein Synthesis. Methods Protoc. 2(4). pii: E80. doi: 10.3390/mps2040080.

104. Jeong D, Klocke M, Agarwal S, Kim J, Choi S, Franco E, Kim J. (2019) Cell-Free Synthetic Biology Platform for Engineering Synthetic Biological Circuits and Systems. Methods Protoc. 2(2). pii: E39. doi: 10.3390/mps2020039.
This work is licensed under Creative Commons Attribution 4.0 License

To Submit Your Article Click Here: Submit Manuscript

DOI: $10.31579 / 2766-2314 / 042$
Ready to submit your research? Choose Auctores and benefit from:

*ast, convenient online submission
*igorous peer review by experienced research in your field
*apid publication on acceptance
* unthors retain copyrights
- immediate, unrestricted online access

At Auctores, research is always in progress.

Learn more www.auctoresonline.org/journals/biotechnology-andbioprocessing 